\title{
A server-side program for delivering experiments with animations
}

\author{
WILLIAM C. SCHMIDT \\ State University of New York, Buffalo, New York
}

\begin{abstract}
A server-sideprogram for animation experiments is presented. The program is capable of delivering an experiment composed of discrete animation sequences in various file formats, collecting a discrete or continuous response from the observer, evaluating the appropriateness of the response, and ensuring that the user is not proceeding at an unreasonable rate. Most parameters of the program are controllable by experimenter-edited text files or simple switches in the program code, thereby minimizing the need for programming to create new experiments. A simple demonstration experiment is discussed and is freely available.
\end{abstract}

The Web is quickly becoming a popular medium for the delivery of psychological experiments (Reips, 2001). For experiments involving graphical sequences of images, experimenters have a wide range of options (see Schmidt, 2001, for an overview) from client-side programming of the animation sequence (i.e., using Authorware, Java, or JScript/Javascript) to using plug-ins or built-in browser support for presenting animations in a particular animation format (i.e., Authorware, Flash, or Quicktime animations require plug-ins, whereas animated GIFs do not).

If one takes the client-side programming approach, additional programming can be done to collect subject responses, and accurate response time dependent measures may be possible. The drawback to relying on client-side programming is that the experimenter is relying on potential subjects to access the experiment with a computer system that has the capability of adequately running the clientside experiment program. Because this requires extra software (i.e., a compatible Java interpreter, the Authorware browser plug-in, activated JScript/Javascript, etc.) or speedier hardware (i.e., Authorware; see Schmidt, 2001), it is likely that some potential subjects may be unable to participate, whereas others who do participate may produce highly variable data owing to software or hardware differences.

The server-side programming approach on the other hand does not allow for highly accurate collection of response time data, although with some server-side programming, many sorts of subject responses can be obtained. The main benefit of this approach is that it relies less on the capabilities of the potential subject's computer, putting the burden of computation instead on the server side of the client-server relationship (see Schmidt, 2000, for a discussion of the capabilities of server-side programs).

Correspondence concerning this article should be addressed to W. C. Schmidt, Department of Psychology, SUNY at Buffalo, Park Hall, Buffalo, NY 14260-4110(e-mail: wcswcs@buffalo.edu).
If the appropriate animation method is used (i.e., one that the subject's computer is configured to display), participation of greater numbers of potential subjects can be achieved. Furthermore, because server-side programming is necessary to accept data from any method of networked experiment delivery, it is highly likely that experimenters will need to gain some knowledge of these skills regardless of the approach they take in implementing their experiment.

The present paper presents a general program for serverside control and delivery of experiments with an animation on each trial followed by the collection of a response. That is, a program runs on the server that delivers a series of Web pages to the client with animations and form elements embedded within. The animation proceeds, and after the user supplies a response, the response is sent back to the server program for processing. The response can be either continuous (clicking somewhere along a scale) or discrete (clicking a particular button or within a particular region).

The server-side program has been designed to allow new experiments to be generated by creating an animation in a graphics package of one's choosing and by specifying the animation uniform resource locator (URL) for each trial in a text file. This approach provides the potential to execute graphical Web experiments without using client-side programming. The collection of user responses may require specific programming, depending on the experiment requirements. Once some paths are updated in the program code and the appropriate files uploaded to the server, the experiment is ready to begin.

\section{Requirements}

In order to use this general server-side experiment program, one needs access to a Web server and must have the ability to edit text files and install and run common gateway interface (CGI) programs (see Schmidt, Hoffman, \& MacDonald, 1997, for an introduction to running a Web server for experimental purposes, and Kieley, 1996, for information about CGI programming). It is assumed 
that the experimenter has basic skills in this domain. The program is written in practical extraction and report language (Perl), a versatile and friendly programming milieu that is popular for writing CGI programs. An excellent, short tutorial for Perl is available on line at http:// www.comp.leeds.ac.uk/Perl/start.html. Free Perl interpreter installers for a variety of operating systems can be located through http://www.perl.com.

Web-based graphics animations or sound files are also required for the use of the present program (see Schmidt, 2001 , for an empirical evaluation of animation presentation accuracy for these and other Web animation methods). The explanation of the creation of such files is beyond the scope of the present work, but it should be noted that animations can be generated in any of a number of different software packages. A great variety of tools for a variety of operating systems are listed at http://desktoppublishing.com/anitools.html.

\section{Overview of the Procedure}

An example experiment can be viewed at http://survey. psy.buffalo.edu:8080/example/consent.html. The source code for the experiment, configuration files, and data files to be referred to can be obtained through the following URL: http://survey.psy.buffalo.edu:8080/example/. The source code is listed in the Appendix for archival purposes and easy reference.

The example experiment presents an informed consent form and then passes the consenting user to some instructions and to a CGI program (example.pl) that delivers an experiment with eight trials. The program is currently written to record information about a user's impression of motion within a line that appears sequentially over time. The animation method used is animated graphic interchange format files (GIFs). This is the only animation method that graphical browsers universally support (at least from Netscape 2.0 and Internet Explorer 1.0 onward), that does not have to be installed or enabled by the user, and that cannot be disabled. One drawback with animated GIFs is that, like most Web animation techniques, their timing accuracy level varies with the speed of the client computer. For GIFs, presentation durations under $300 \mathrm{msec}$ per frame become highly variable (Schmidt, 2001).

In the paradigm implemented, both direction and strength of motion are relayed via a mouse click on an image. The image is a continuous scale that clearly demarks areas pertaining to leftward motion, rightward motion, and no motion. The coordinates of the user's click on the scale are passed back to the server-side program. The program extracts the coordinates of the click and classifies the response according to one of three categories (leftward, rightward, and no motion). The exact value along the scale indicates magnitude or strength of the motion experience. The image and the program could be modified so that any sort of classification could be used. For instance, the image could be a number of labeled buttons or a single continuous strip. Alternatively, with extra programming, the form elements implemented by any browser could be used to acquire responses (see the Alternative Input and Output section).

After submission of the data from a single trial, the program processes the data submitted and presents the next trial. If the subject responds to any trial at a rate that has been specified by the experimenter as suggesting that he or she is not completing the task in a serious manner, the response is not accepted and a new trial is presented. This feature is controlled by specifying the minimum acceptable duration of a trial (including client-server communication time), so setting this duration to zero disables trial length constraints completely.

Once the experiment trials are complete, the subject is automatically redirected to another URL. In the case of the example, the subject is shown a closing page thanking him or her for participating and supplying more information about the study. As a result of the redirection feature, several experiments can be chained together to redirect the subject from one set of trials to another, thereby allowing for blocks of trials or for a diverse set of tasks within a single experimental session.

\section{Program Output}

The program writes a record of the experimental session to a comprehensive data file upon each subject's completion of the experiment. Until a subject has completed the experiment, a record of his or her responses and the order of the trials are kept in separate files in the program directory, named by their Internet protocol (IP) number. The _permuted file contains the order in which trials are to be presented, and the _responses file contains completed trials and their associated responses. These files are removed once a subject has completed the experiment, and the data are transferred to the experiment data file. Data from partially completed experiments can be recovered by examining these files. One caveat with regard to this method is that subjects with dynamic IP numbers may have some problems completing the experiment should their IP numbers change while they are participating.

The format of the summary data file (see the file named "exampledata" on line) is tab delimited so that it can easily be imported into most statistical packages. First, some identifying information, such as the subject's IP number and browser type, is stored, along with the date and time of completion. Next, for each trial in the order presented to the subject, come the trial number, the animation URL, the location on the imagemap that was clicked, and the response category that was assigned. For instance, the entry "http://survey.psy.buffalo.edu:8080/gifs/leftdrawnILM. gif 342 " indicates that the leftdrawnILM animation produced a response of " 2 " (leftward) and a click on the scale at Pixel 34 (on a 512-pixel scale, the width of the imagemap GIF). After each trial has been recorded, some summary information about each unique animation appears. This summary information is the data that are likely to be used in analyses.

The animation summary information appears in the data file in alphabetical order, so that any desired output ordering can be achieved by strategically naming the an- 
imations in order to control the summary information position in the data file (i.e., 1.gif will appear before 2.gif). If systematic naming of stimulus files is of concern to the experimenter, random naming techniques are available (see Reips, 2001).

The example experiment counts the number of trials on which a subject contributed data for the given animation, computes the frequency with which each response category was selected for the animation, and then averages the response magnitudes. An example entry of the summary information is "http://survey.psy.buffalo.edu:8080/gifs/ leftdrawnILM.gif 440060.5 ," indicating that, for the leftdrawnILM animation, four trials were involved. All four times, leftward was selected, with no motion and rightward being selected zero times. Finally, the average of the response magnitudes was 60.5. This is just an example of the type of information that can be computed by the program; experimenters may wish to customize the program to generate other sorts of summary information, depending on their experimental designs, or they may wish to compute such information from the raw data after the experiment has been completed.

\section{Creating an Experiment}

To create an experiment, the user must (1) edit a file that contains a listing of trials in the experiment, (2) create animation files, (3) modify switches or parameters in the program, (4) create lead-in and lead-out Web pages, (5) upload the files to the Web server, and (6) test and debug. The file that contains a listing of trials (the "trial file") is a text file. For each trial, there are two columns. The first column is simply the trial number, and the second column is the URL to the animation. For instance, the trial:

\section{1 http://survey.psy.buffalo.edu:8080/gifs/leftdrawn.gif}

is trial number one and would present an animation called "leftdrawn.gif". The animations were drawn in Microsoft Powerpoint and made into animated GIFs by using the freely available program Gif Builder on the Macintosh.

The program file is also a text file. Near the beginning of the program file are a number of modifiable switches or lines of code. The assignment to \$TITLE is the text that appears in the browser's title bar on each trial. The assignments to \$trialfilename and \$datafilename are the names of files in the same directory as the program that contains the trials and the data, respectively. The assignment to \$randomize indicates whether the trials are to be presented in random order (a setting of 1) or in the order listed in the trial file (a setting of 0 ). The value assigned to \$trialduration reflects the minimum number of seconds from the time the trial is delivered until the server receives the subject's response. If the subject responds before this duration has elapsed, a message to "slow down" is issued. Note that this duration includes Internet travel time, as well as the subject's response time. The server path assigned to \$path informs the program where to place the data file and temporary files that hold information about a particular user's randomization and responses.
The program is currently set to deliver animations specified by the $<$ IMG $\operatorname{src}=\ldots></$ IMG $>$ tag. To modify this in order to deliver a different type of animation, search for the IMG tag in the program and replace it with the appropriate method of calling your animation. A flash animation, for instance, would use the text $\angle$ EMBED $\mathrm{src}=\ldots></$ EMBED $>$. Auditory samples could be played, as could any animation method that presents information within the browser window.

\section{Alternative Input and Output}

Alternative responses could be achieved in several ways. The easiest would be to replace the GIF representing the current motion scale with one partitioned into separate regions (i.e., buttons) corresponding to responses of interest to the paradigm of one's choice. Modify the program code by placing a new URL after the symbol "-src=>" in the program code and, possibly, by modifying the height or width values in the neighboring lines. The width and height of the image, in pixels, correspond to the pixel position that the browser will return regarding a user's click. Next, modify the section of the program code that deals with processing the response (following \# RECORD and \# FINISHED in the source code of the Appendix) so that it correctly classifies the subject's response on the basis of the clicked coordinates. This code currently tests for specific coordinates in the imagemap corresponding to demarcations of the different response categories.

Alternative user interfaces containing any of the form elements that modern browsers support are possible. The use of buttons is very easy in CGI Perl programming, making any discrete data acquisition task relatively straightforward (examine example CGI programming in the examples of Lincoln Stein, the author of the Perl CGI module [http://stein.cshl.org/WWW/software/CGI/cgi_docs. $\mathrm{html}]$ ). Incorporating such example code into the current framework is relatively straightforward and would be handled in place of the code that currently tests the imagemap input (following \# RECORD) and in the code that presents a trial (following \# NOT FINISHED).

Response categories can be changed by modifying the code wherever the variables \$leftmotion, \$rightmotion, or \$nomotion are found (following \# FINISHED). Currently, the code checks where the user clicked and categorizes the response on the basis of that click. Depending on the design and purposes, in other experiments, one may wish to keep track of different response categories or to summarize the data in a different fashion. Of course, in order to generate a different sort of data summary, some custom programming will be required.

\section{Summary}

A server-side program for controlling simple graphical experiments with discrete trials and, preferably, a nonresponse time dependent measure is presented. The program can be used to deliver any Web-based file format embeddable within the browser window (i.e., any animation or sound format) and accepts discrete or continuous 
responses. The program can be expanded for use in other experimental paradigms with some programming effort or can be used in a similar fashion with minimal changes.

\section{REFERENCES}

KIELEY, J. M. (1996). CGI scripts: Gateways to World-Wide Web power. Behavior Research Methods, Instruments, \& Computers, 28, 165169.

ReIPS, U.-D. (2001). The Web Experimental Psychology Lab: Five years of data collection on the internet. Behavior Research Methods, Instruments \& Computers, 33, 201-211.

Schmidt, W. C. (2000). The server-side of psychology Web experiments. In M. H. Birnbaum (Ed.), Psychological experiments on the Internet, pp. 285-310. San Diego: Academic Press.

SchmidT, W. C. (2001). Presentation accuracy of Web animation methods. Behavior Research Methods, Instruments \& Computers, 33, 187-200.

Schmidt, W. C., Hoffman, R., \& MacDonald, J. (1997). Operate your own World-Wide Web server. Behavior Research Methods, Instruments \& Computers, 29, 189-193. 


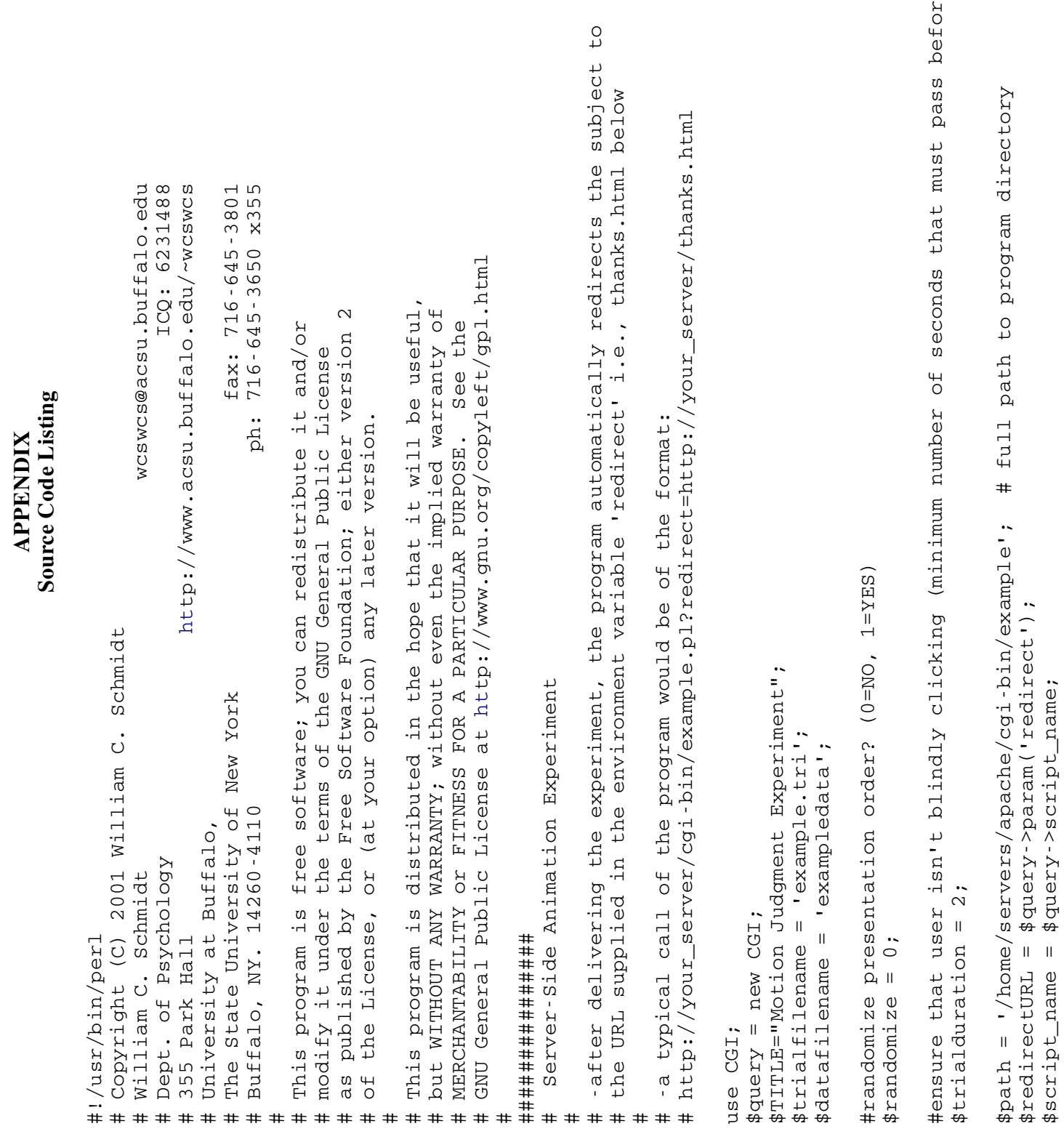



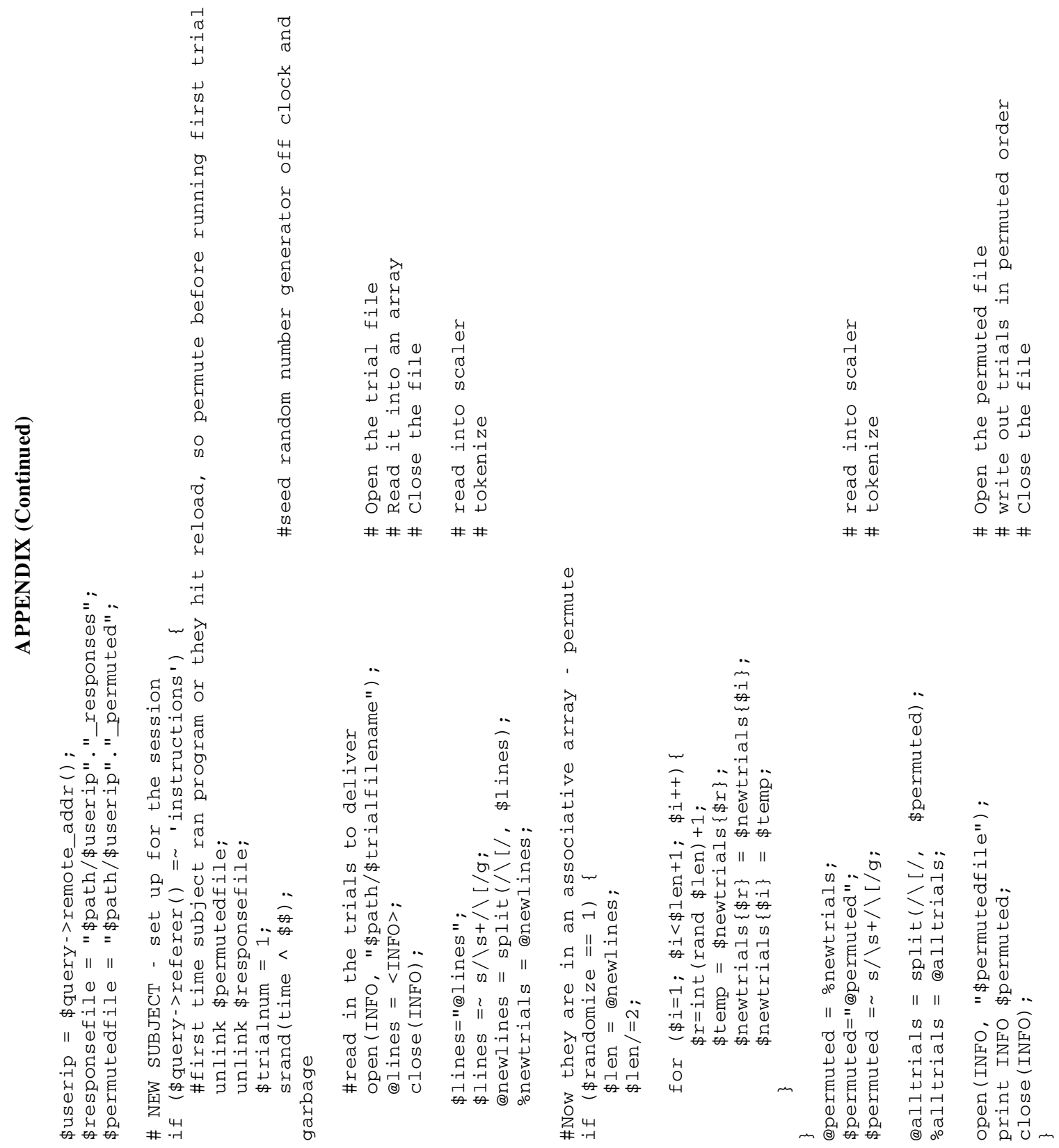


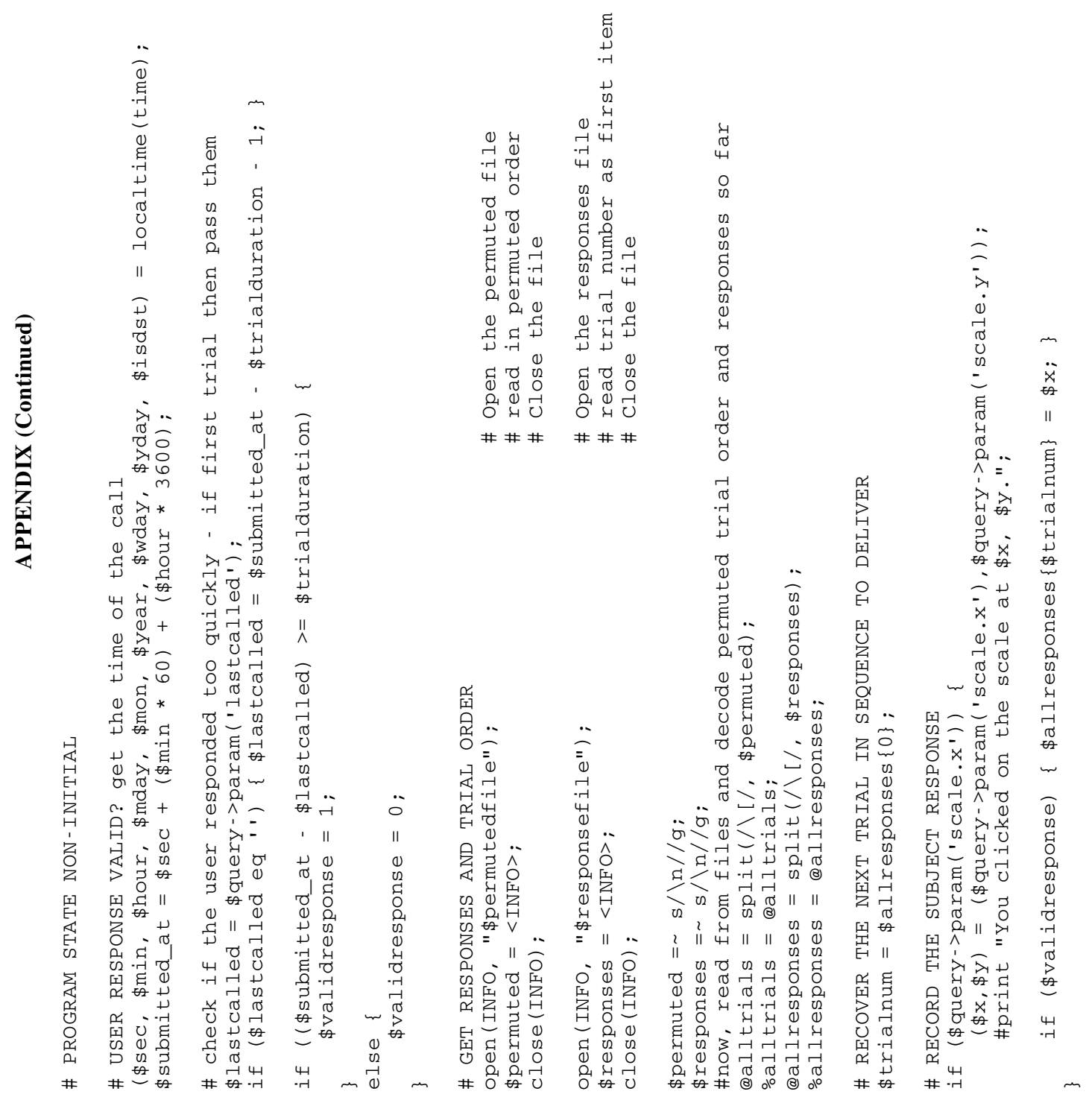



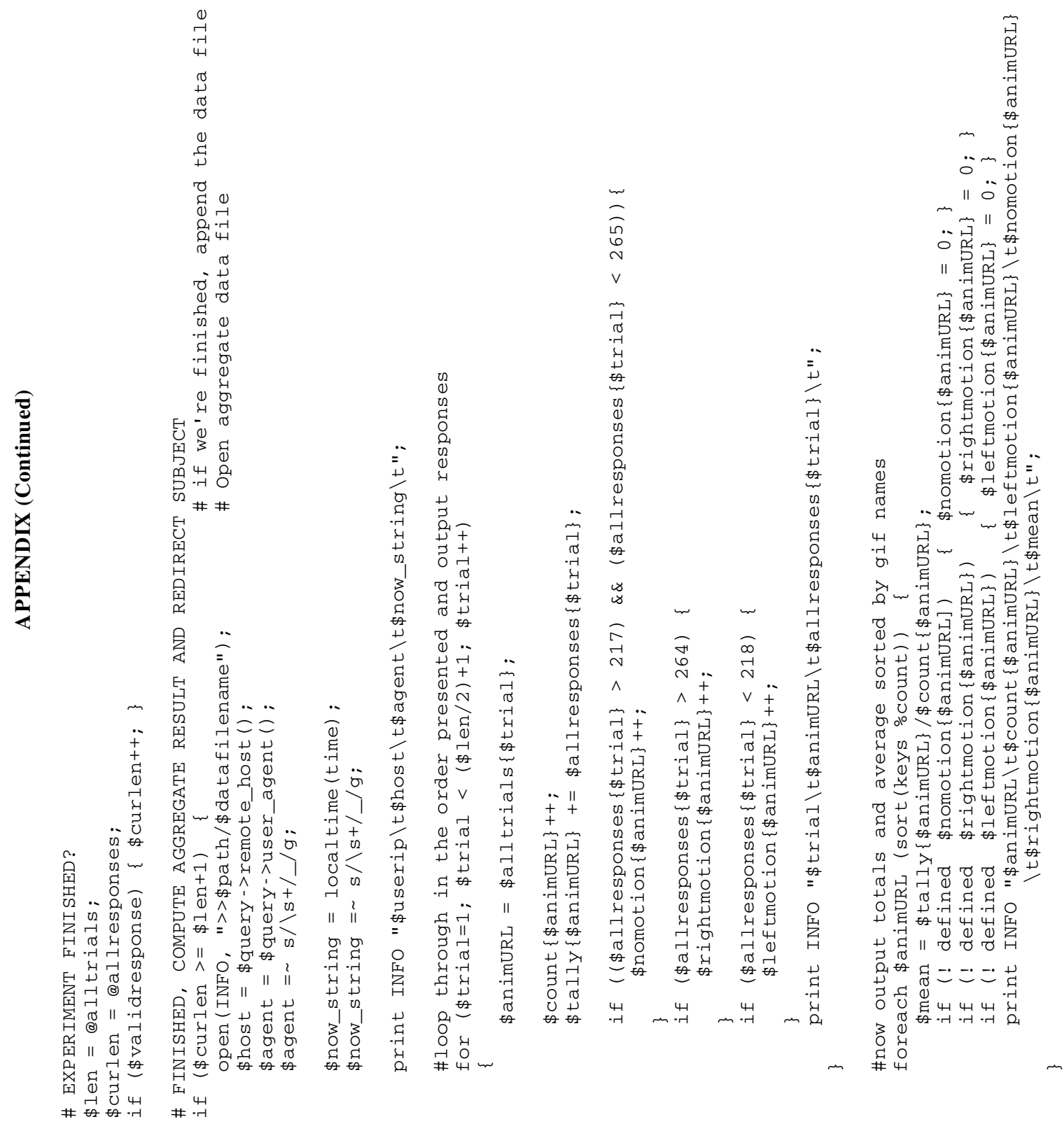

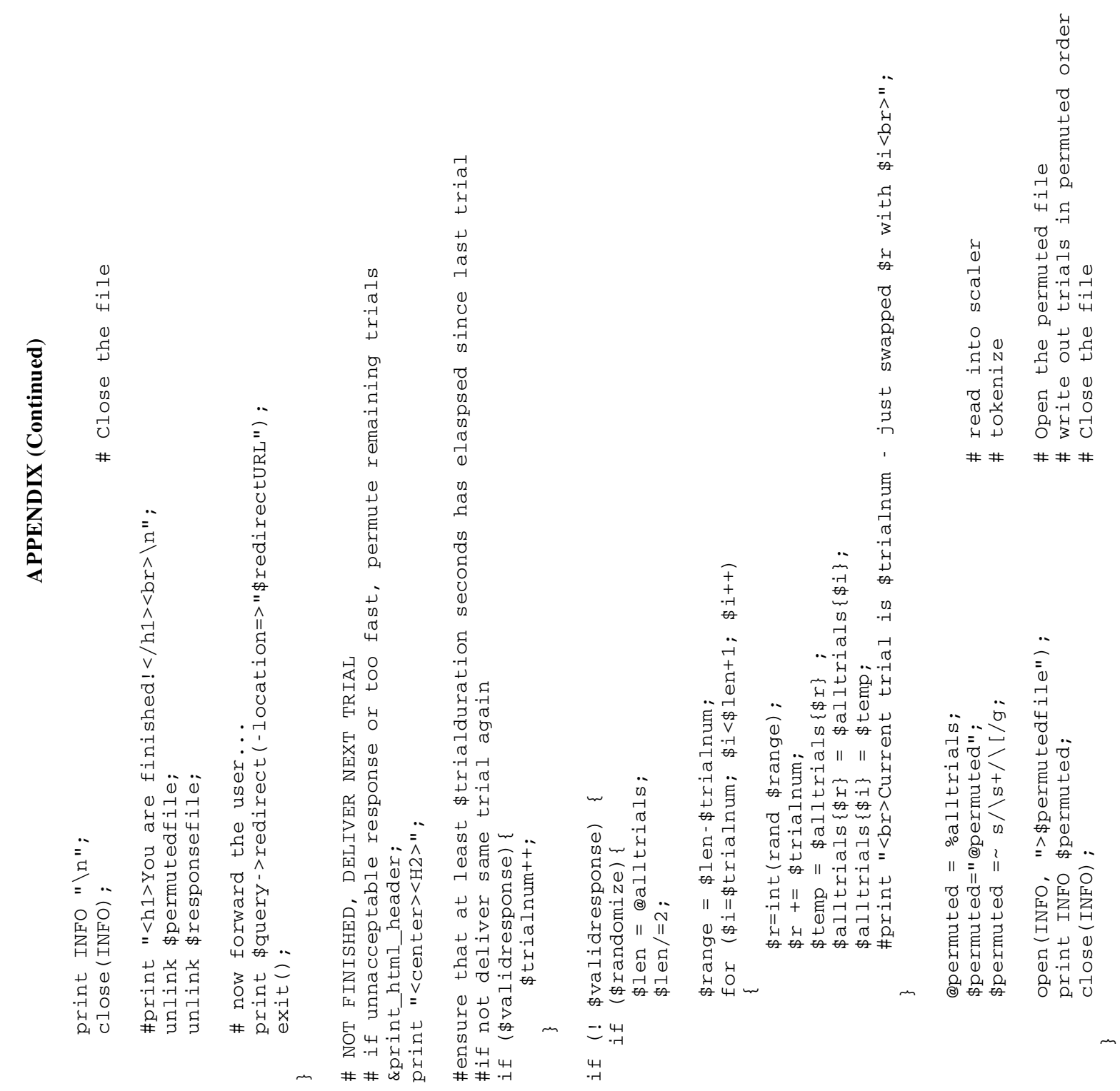


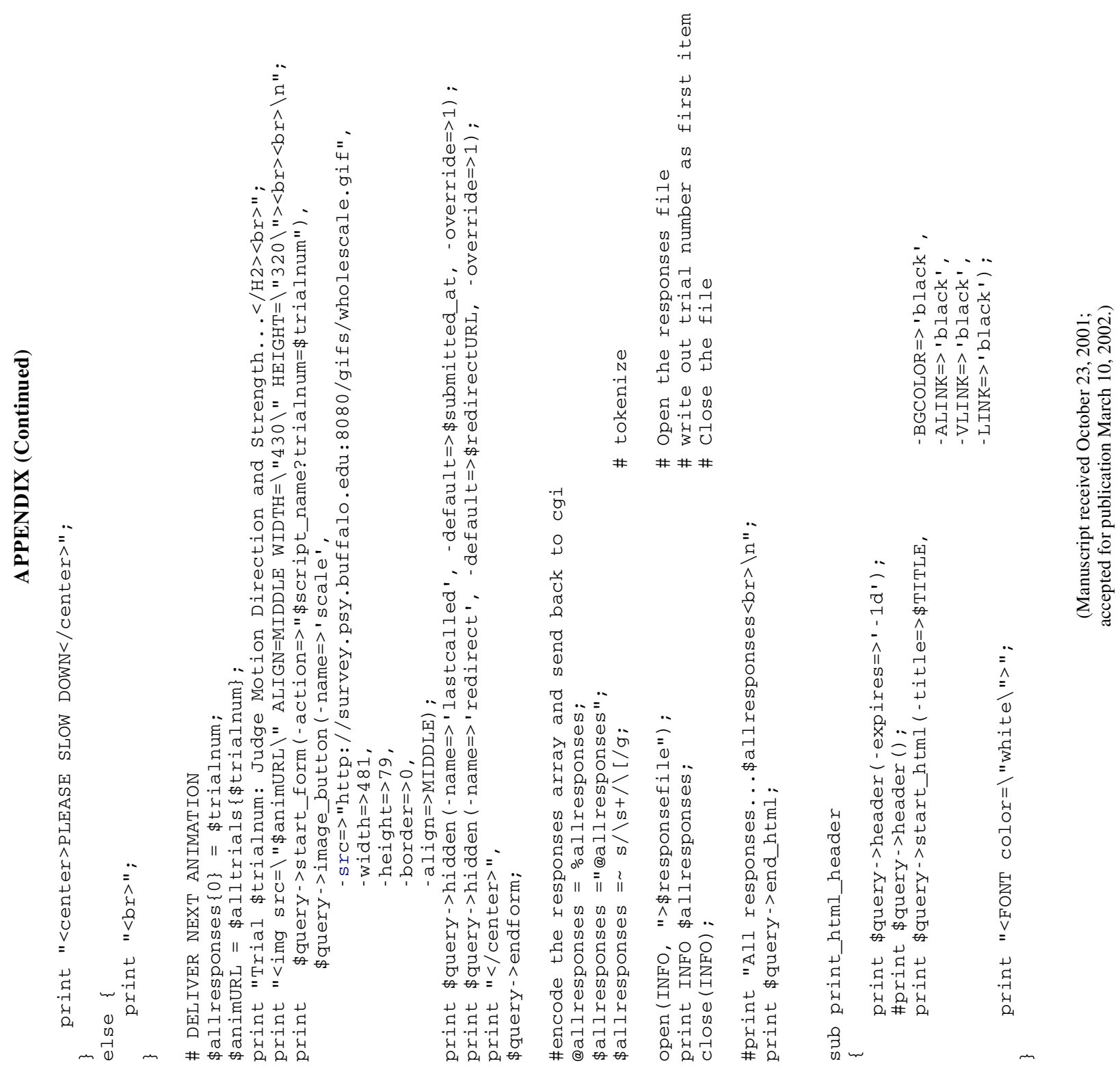

